\title{
A Spherical Cap Harmonic Model of the Satellite Magnetic Anomaly Field Over China and Adjacent Areas
}

\author{
Zhen-chang $\mathrm{AN}^{1}$, Shi-zhuang $\mathrm{MA}^{1}$, Dong-hai $\mathrm{TAN}^{1}$, D. R. BARRAClOUGH ${ }^{2}$, \\ and D. J. KERRIDGE ${ }^{2}$ \\ ${ }^{1}$ Institute of Geophysics, Chinese Academy of Sciences, 100101, Beijing, China \\ ${ }^{2}$ British Geological Survey, Murchison House, West Mains Road, Edinburgh EH9 3LA, U.K. \\ (Received October 18, 1991; Revised January 30, 1992)
}

\begin{abstract}
Using Magsat data over China and adjacent areas (between latitudes $10^{\circ} \mathrm{N}$ and $60^{\circ} \mathrm{N}$ and longitudes $70^{\circ} \mathrm{E}$ and $140^{\circ} \mathrm{E}$ ), a spherical cap harmonic model describing the three-dimensional structure of the satellite magnetic anomaly field has been derived. The pole of the cap was at $\left(35^{\circ} \mathrm{N}, 105^{\circ} \mathrm{E}\right)$, its half-angle was $35^{\circ}$ and a maximum index of 12 was used. The maximum spherical harmonic degree corresponding to this index is 31.6, giving a minimum wavelength, at the Earth's surface, of $1266 \mathrm{~km}$. From this model and the International Geomagnetic Reference Field (IGRF), a model of the total intensity anomaly field $(\Delta F)$ was derived. The satellite magnetic anomalies $(\Delta X, \Delta Y, \Delta Z, \Delta F)$ at an altitude of $400 \mathrm{~km}$ were calculated and the corresponding magnetic anomaly charts have been drawn.
\end{abstract}

\section{Introduction}

Satellite magnetometer observations permit the characterisation of magnetic signatures for lithospheric regions hundreds or even thousands of kilometres in extent. Such information is not readily obtainable from conventional aeromagnetic surveys. Regions of this size, identified and characterised on a global basis, provide useful information for deciphering the Earth's history, including ancient and contemporary geodynamics, for the separation of different parts of the lithosphere into resource provinces and for the numerical modelling of lithospheric processes.

The Magsat data includes information on the direction and strength of the geomagnetic field. The data have been used to compile hand-drawn charts of satellite magnetic anomalies (AN et al., 1992b). These charts do not, however, take account of altitude variations in the data, nor do they satisfy physical constraints that arise from the theory of potential fields. In addition, such hand-drawn charts cannot provide analytical expressions for the magnetic anomalies.

In order to model the three-dimensional structure of the satellite magnetic anomaly field, accounting for the distribution of the data in altitude and the potential field constraints on the curl and the divergence of the internal field, we have used the technique of spherical cap harmonic analysis as described by HAINES (1985).

This paper presents our three-dimensional model of satellite magnetic anomalies in China and adjacent areas together with a preliminary discussion of some features of the anomaly field. 


\section{Data Processing}

The data used in this study were taken from the Magsat Investigator-B magnetic tapes (LANGEL et al., 1981). Data having latitudes between $10^{\circ} \mathrm{N}$ and $60^{\circ} \mathrm{N}$ and longitudes between $70^{\circ} \mathrm{E}$ and $140^{\circ} \mathrm{E}$ were selected. Although the twilight orbits flown by Magsat were chosen to minimise the effects of ionospheric currents, most researchers who have used Magsat data for studying magnetic anomalies have found that passes where the $K_{p}$ index was greater than $2_{0}$ are difficult to use because of difficulties in modelling ionospheric and magnetospheric fields adequately. Therefore, only data from passes with $K_{p} \leq 2_{0}$ were used in this study. If the total number of points for any pass was less than 10 the data from that pass were rejected. After applying these selection criteria there were 397 dawn passes and 427 dusk passes. The combined data set contained almost 100000 observed values of the total intensity and the three components of the vector field.

The magnetic field observed by Magsat is due to electric currents flowing in the Earth's core and in the ionosphere and the magnetosphere; to currents in the crust and mantle induced by time variations in the ionospheric and magnetospheric fields; and to the magnetisation of crustal rocks. In order to isolate the crustal anomaly fields, the core (or main) field, the ionospheric and magnetospheric fields and the induced fields must be subtracted from the Magsat data. The steps involved in the extraction of the crustal field data will now be described.

\subsection{Removal of the main, external and induced fields}

We used GSFC(12/83), the spherical harmonic model of the main field and its secular variation at epoch 1980.0 derived by LANGEL and ESTES (1985), to remove the main, external and induced fields from the selected Magsat data. This model includes main-field coefficients (up to and including degree $(n)$ and order $(m) 13$ ), secular-variation coefficients (up to and including $n=m=10$ ), external-field coefficients (up to and including $n=m=1$ ), and the effects of induced currents on the internal field coefficient $g^{0}{ }_{1}$.

For the location $(r, \theta, \lambda)$ and time of observation $(t)$ of each data point, the main, external and induced fields were calculated, using the GSFC(12/83) model, and were subtracted from the Magsat data:

$$
\Delta \boldsymbol{B}_{\mathrm{c}}=\boldsymbol{B}_{\mathrm{o}}-\left(\boldsymbol{B}_{\mathrm{m}}+\boldsymbol{B}_{\mathrm{e}}\right)
$$

where $\boldsymbol{B}_{\mathrm{o}}$ is the Magsat observed value, $\boldsymbol{B}_{\mathrm{m}}$ the main field (including the induced field) calculated from the GSFC(12/83) model, $\boldsymbol{B}_{\mathrm{e}}$ the external field computed from this same model, and $\Delta \boldsymbol{B}_{\mathrm{c}}$ the initial magnetic anomaly value.

\subsection{Quadratic fit in latitude to the initial magnetic anomalies for each pass}

The initial magnetic anomaly values are not consistent for some geographically adjacent passes flown at different times. These inconsistencies have been attributed to a failure to account for time variations of the long-wavelength external fields due to variations in the ring current and large-scale changes in the size of the magnetosphere. In order to improve the passto-pass consistency we have used a second-degree polynomial in latitude to fit the initial magnetic anomaly field for each pass: 


$$
\Delta B(i)=A_{0}+A_{1} \Delta \phi(i)+A_{2}[\Delta \phi(i)]^{2}
$$

where $\Delta \phi(i)=\phi(i)-\phi(1) ; \phi(1)$ is the latitude of the first observed point of each pass; $\phi(i)$ is the latitude of the $i$ th point of this same pass; $\boldsymbol{A}_{0}, \boldsymbol{A}_{1}, \boldsymbol{A}_{2}$ are the coefficients of the polynomials which are determined by using the method of least squares to fit the initial anomaly values $\Delta \boldsymbol{B}_{\mathrm{c}}$ for each pass; and $\Delta \boldsymbol{B}(i)$ is the value computed from the polynomial fit.

Such second-degree polynomials were derived for each pass. Using these polynomials, the fitted value $\Delta \boldsymbol{B}(i)$ was calculated at each observation point for the corresponding pass and this value was subtracted from the initial anomaly value $\Delta \boldsymbol{B}_{\mathrm{c}}(i)$ to give the final anomaly $\Delta \boldsymbol{B}_{\mathrm{r}}(i)$ :

$$
\Delta \boldsymbol{B}_{\mathrm{r}}(i)=\Delta \boldsymbol{B}_{\mathrm{c}}(i)-\Delta \boldsymbol{B}(i)
$$

\subsection{Rejection of spurious data}

There may be some occasional spurious data in the residual values. In order to eliminate these the mean, $\left\langle\Delta \boldsymbol{B}_{\mathrm{r}}\right\rangle$, and standard deviation, $\sigma$, were calculated for each pass. Then, if

$$
\left|\Delta \boldsymbol{B}_{\mathrm{r}}(i)-\left\langle\Delta \boldsymbol{B}_{\mathrm{r}}\right\rangle\right|>4 \sigma,
$$

the corresponding anomaly value, $\Delta \boldsymbol{B}_{\mathrm{r}}(i)$, was rejected. In other words, any anomaly value differing from the mean value for its pass by more than four times the standard deviation for that pass was deleted. A new quadratic fit was then derived, new crustal anomaly values were calculated and the procedure was repeated until no further points were rejected.

\subsection{Creation of equal-area grid}

There were approximately 100000 data points remaining after these selection and rejection procedures had been carried out. A grid consisting of cells each equal in area to a $1^{\circ}$ $\times 1^{\circ}$ cell on the equator in spherical cap coordinates was used and mean values of the positions $(r, \theta, \phi)$ and the anomaly values $(\Delta X, \Delta Y, \Delta Z)$ were derived from the observations lying in each such cell.

\section{Mathematical Method}

The three-dimensional model of the satellite anomalies $\Delta X, \Delta Y, \Delta Z$ was expressed directly in terms of spherical cap coordinates:

$$
\begin{gathered}
\Delta X=\sum_{k=1}^{K_{\max }} \sum_{m=0}^{k}(a / r)^{n_{k}(m)+2}\left(g_{k}^{m} \cos m \Lambda+h_{k}^{m} \sin m \Lambda\right) \frac{\mathrm{d} P_{n_{k}(m)}^{m}(\cos \psi)}{\mathrm{d} \psi}, \\
\Delta Y=\sum_{k=1}^{K_{\max }} \sum_{m=1}^{k} \frac{m}{\sin \psi}(a / r)^{n_{k}(m)+2}\left(g_{k}^{m} \sin m \Lambda-h_{k}^{m} \cos m \Lambda\right) P_{n_{k}(m)}^{m}(\cos \psi), \\
\Delta Z=-\sum_{k=0}^{K_{\max }} \sum_{m=0}^{k}\left(n_{k}(m)+1\right)(a / r)^{n_{k}(m)+2}\left(g_{k}^{m} \cos m \Lambda+h_{k}^{m} \sin m \Lambda\right) P_{n_{k}(m)}^{m}(\cos \psi),
\end{gathered}
$$


Table 1. Values of the spherical cap harmonic coefficients $g^{m}{ }_{k}$ and $h^{m}{ }_{k}$. The spherical cap half-angle is $35^{\circ}$.

\begin{tabular}{|c|c|c|c|c|c|c|c|}
\hline $\mathrm{k}$ & $m$ & $g_{k}^{m}(\mathrm{nT})$ & $h_{k}^{m}(\mathrm{nT})$ & $k$ & $m$ & $g_{k}^{m}(\mathrm{nT})$ & $h_{k}^{m}(\mathrm{nT})$ \\
\hline 0 & 0 & -0.7 & & 9 & 0 & -29.7 & \\
\hline 1 & 0 & 16.0 & & 9 & 1 & -643.7 & -72.5 \\
\hline 1 & 1 & -18.3 & -5.8 & 9 & 2 & 312.3 & -41.4 \\
\hline 2 & 0 & -55.8 & & 9 & 3 & 311.0 & 142.6 \\
\hline 2 & 1 & 100.4 & 30.1 & 9 & 4 & -175.6 & -122.7 \\
\hline 2 & 2 & -5.3 & 7.8 & 9 & 5 & -19.4 & -28.4 \\
\hline 3 & 0 & 134.6 & & 9 & 6 & 21.5 & 44.8 \\
\hline 3 & 1 & -280.2 & -80.8 & 9 & 7 & -26.5 & 8.5 \\
\hline 3 & 2 & 38.9 & -33.7 & 9 & 8 & -3.3 & 7.6 \\
\hline 3 & 3 & 30.5 & 1.5 & 9 & 9 & 1.0 & 3.0 \\
\hline 4 & 0 & -222.0 & & 10 & 0 & 44.7 & \\
\hline 4 & 1 & 599.2 & 161.0 & 10 & 1 & 289.0 & 24.8 \\
\hline 4 & 2 & -116.0 & 79.9 & 10 & 2 & -175.0 & 3.9 \\
\hline 4 & 3 & -113.6 & -13.2 & 10 & 3 & -147.8 & -85.2 \\
\hline 4 & 4 & 25.8 & 17.2 & 10 & 4 & 99.5 & 68.0 \\
\hline 5 & 0 & 277.1 & & 10 & 5 & 3.9 & 17.9 \\
\hline 5 & 1 & -954.7 & -233.9 & 10 & 6 & -13.8 & -33.0 \\
\hline 5 & 2 & 245.0 & -137.0 & 10 & 7 & 22.8 & -7.9 \\
\hline 5 & 3 & 249.0 & 44.4 & 10 & 8 & 1.3 & -6.1 \\
\hline 5 & 4 & -80.0 & -59.2 & 10 & 9 & 0.5 & -4.4 \\
\hline 5 & 5 & -5.3 & -7.4 & 10 & 10 & -0.3 & -1.4 \\
\hline 6 & 0 & -258.8 & & 11 & 0 & -25.0 & \\
\hline 6 & 1 & 1224.0 & 261.0 & 11 & 1 & -87.1 & -6.4 \\
\hline 6 & 2 & -372.7 & 170.7 & 11 & 2 & 64.3 & 6.2 \\
\hline 6 & 3 & -406.2 & -98.2 & 11 & 3 & 45.9 & 35.2 \\
\hline 6 & 4 & 152.9 & 116.1 & 11 & 4 & -34.3 & -24.4 \\
\hline 6 & 5 & 18.8 & 17.8 & 11 & 5 & 2.4 & -8.0 \\
\hline 6 & 6 & -5.5 & -11.0 & 11 & 6 & 4.3 & 14.1 \\
\hline 7 & 0 & 161.7 & & 11 & 7 & -12.1 & 5.2 \\
\hline 7 & 1 & -1258.9 & -226.4 & 11 & 8 & 1.1 & 3.0 \\
\hline 7 & 2 & 446.1 & -156.3 & 11 & 9 & -1.7 & 3.7 \\
\hline 7 & 3 & 492.9 & 150.0 & 11 & 10 & 0.3 & 0.5 \\
\hline 7 & 4 & -214.0 & -161.5 & 11 & 11 & 1.7 & -0.6 \\
\hline 7 & 5 & -31.8 & -28.9 & 12 & 0 & 6.7 & \\
\hline 7 & 6 & 13.9 & 27.9 & 12 & 1 & 11.8 & 1.1 \\
\hline 7 & 7 & -7.2 & 2.3 & 12 & 2 & -12.9 & -2.9 \\
\hline 8 & 0 & -48.5 & & 12 & 3 & -6.4 & -7.4 \\
\hline 8 & 1 & 1015.6 & 146.1 & 12 & 4 & 5.7 & 4.7 \\
\hline 8 & 2 & -425.0 & 104.0 & 12 & 5 & -1.4 & 1.8 \\
\hline 8 & 3 & -450.4 & -169.0 & 12 & 6 & -0.2 & -3.1 \\
\hline 8 & 4 & 226.4 & 165.0 & 12 & 7 & 2.9 & -1.7 \\
\hline 8 & 5 & 32.7 & 32.5 & 12 & 8 & -1.0 & -0.4 \\
\hline 8 & 6 & -22.1 & -43.0 & 12 & 9 & 1.3 & -1.6 \\
\hline 8 & 7 & 18.5 & -5.5 & 12 & 10 & -0.1 & 0.0 \\
\hline \multirow[t]{2}{*}{8} & 8 & 3.2 & -6.0 & 12 & 11 & -1.2 & 0.0 \\
\hline & & & & 12 & 12 & -0.5 & -0.5 \\
\hline
\end{tabular}


where $\Delta X, \Delta Y$, and $\Delta Z$ are defined with respect to the spherical cap coordinates; $r, \psi, \Lambda$ are the radius, colatitude and longitude in spherical cap coordinates, respectively; $a$ is the radius of a reference sphere; $K_{\max }$ is the maximum value of the index $k ; g^{m}{ }_{k}$ and $h^{m}{ }_{k}$ are the model coefficients, of which there are $\left(K_{\max }+1\right)^{2} ; P_{n_{k}(m)}^{m}(\cos \psi)$ is the associated Legendre function of integral order $m$ and non-integral degree $n_{k}(m)$. The values for the degree $n_{k}(m)$ are the roots of the equations

$$
P_{n}^{m}\left(\cos \psi_{0}\right)=0, \quad(k-m) \text { odd }
$$

and

$$
\frac{\mathrm{d} P_{n}^{m}\left(\cos \psi_{0}\right)}{\mathrm{d} \psi}=0, \quad(k-m) \text { even }
$$

The conventional geographical coordinate system must be transformed into the spherical cap coordinate system in which the pole of the cap is at $\left(35^{\circ} \mathrm{N}, 105^{\circ} \mathrm{E}\right)$ and the half-angle of the cap is $35^{\circ}$ as described by AN et al. (1992a). The model coefficients were determined by the method of linear least squares using the gridded values (see Subsection 2.4) as input. They are listed in Table 1.

In order to compute the scalar magnetic anomaly $\Delta F$ from the Magsat vector data, we used the expression:

$$
\Delta F=\frac{X}{F} \Delta X+\frac{Y}{F} \Delta Y+\frac{Z}{F} \Delta Z
$$

where $\Delta X, \Delta Y$ and $\Delta Z$ were computed from Eqs. (1), (2) and (3), and $X, Y, Z$ and $F$ were calculated from DGRF 1980 (BARRACLOUGH, 1987).

In the current study, $K_{\max }=12$ and the spherical cap half-angle is $35^{\circ}$. This results in a model with 169 coefficients. The model has a maximum spherical harmonic degree of 31.6 corresponding to a shortest wavelength of $1266 \mathrm{~km}$ at the Earth's surface.

\section{Results and Discussion}

Contour charts of $\Delta X, \Delta Y, \Delta Z$ and $\Delta F$ for the satellite magnetic anomaly field at an altitude of $400 \mathrm{~km}$ are shown in Figs. 1, 2, 3 and 4, respectively.

As shown in Fig. 4 , the $\Delta F$ anomalies show an approximately south-west to north-east trend, similar to that shown by the Bouguer gravity anomalies in China (YIN, 1980), and can be divided into four belts of alternating sign, as follows.

(1) The Chinese south-east coastal negative anomaly belt, in which the intensity varies from 0 to $-3 \mathrm{nT}$.

(2) The Songlia-Yangtze-Myanmar (Burma) positive anomaly belt, in which there are four positive anomaly foci. The first, $5 \mathrm{nT}$ in size, is in Myanmar (Burma); the second, of intensity $5 \mathrm{nT}$, is in the region of the Yangtze Platform; the third, $4 \mathrm{nT}$ in size, is in the Songlia area; and the fourth $(3 \mathrm{nT})$ is in southern Japan. 


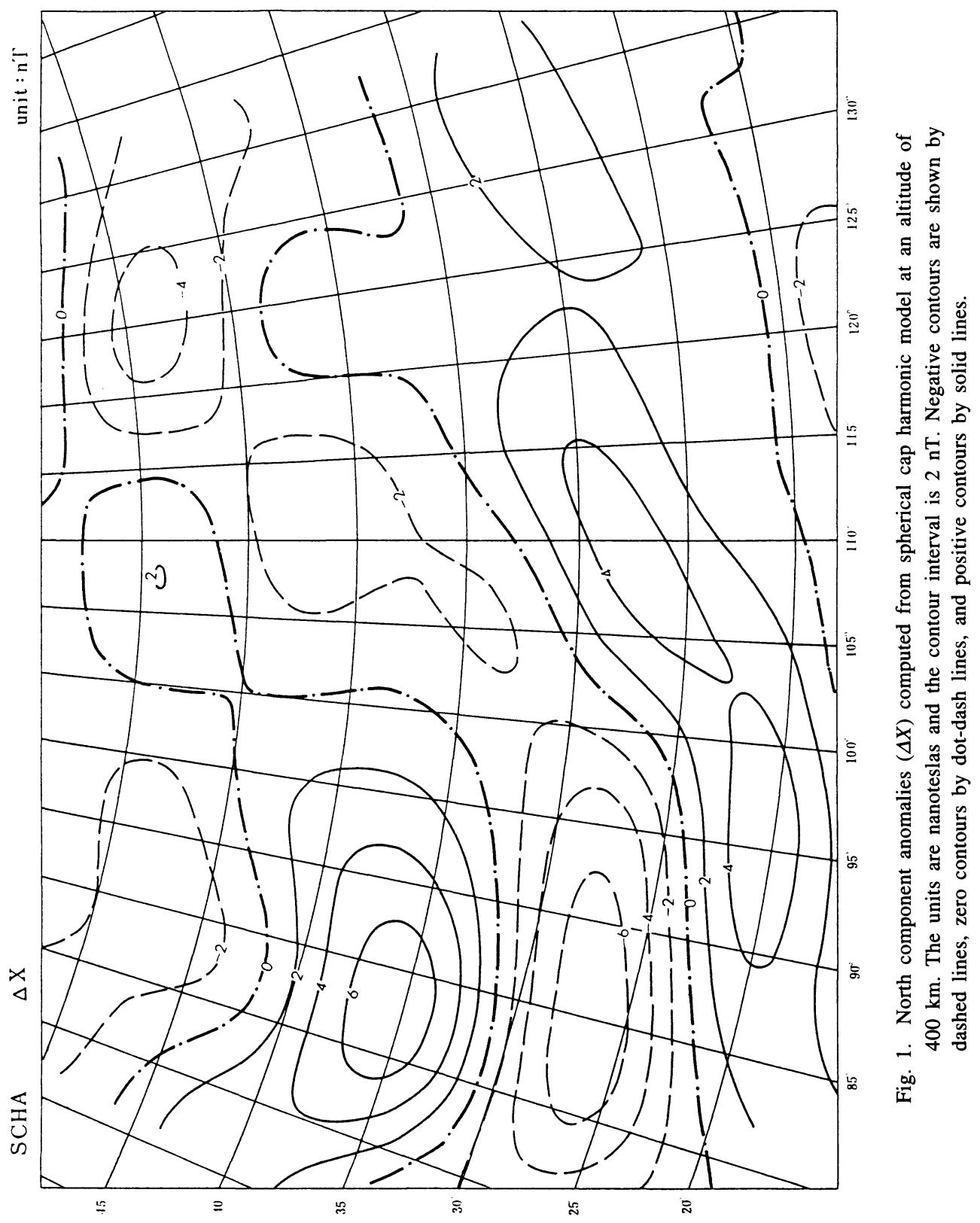




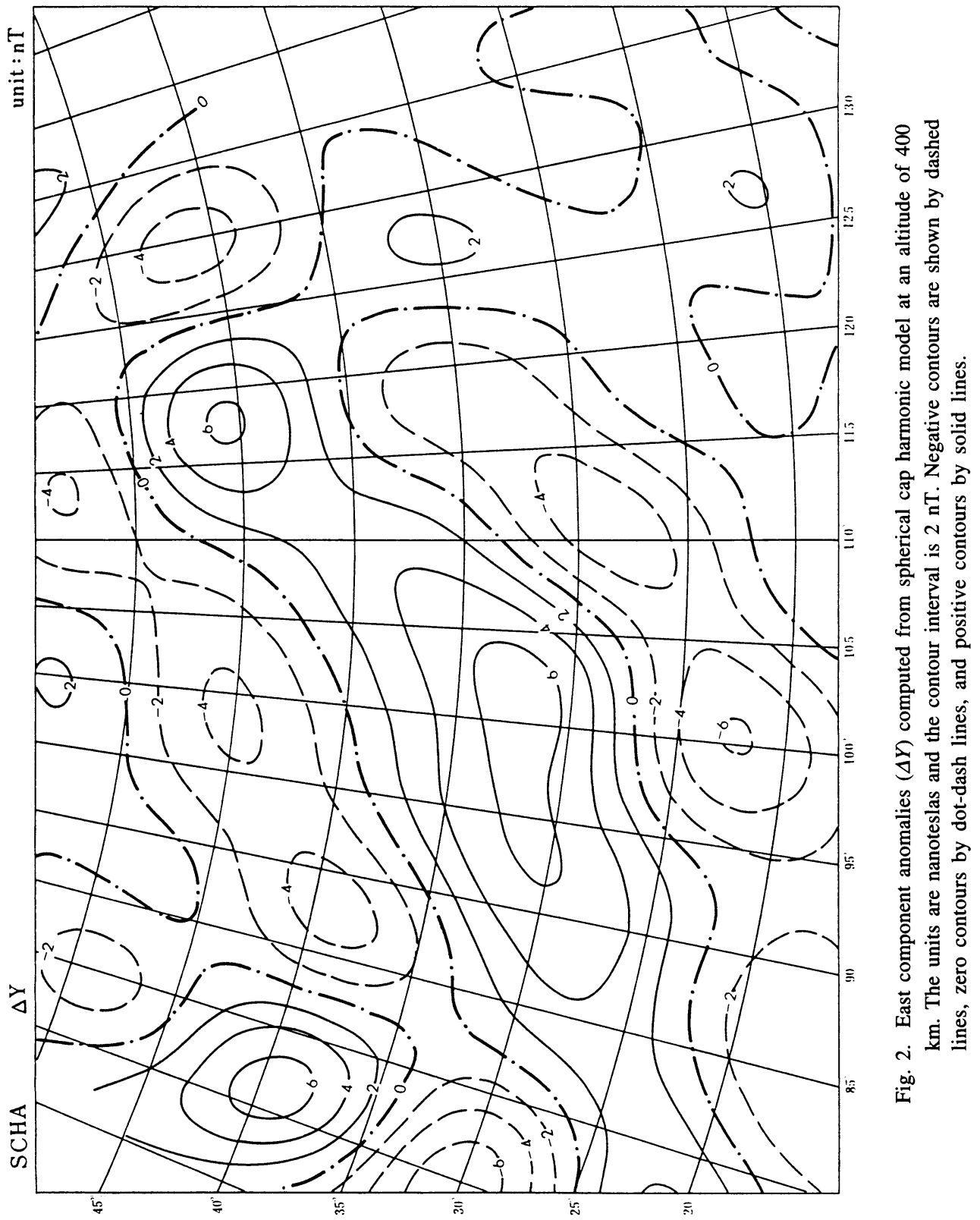




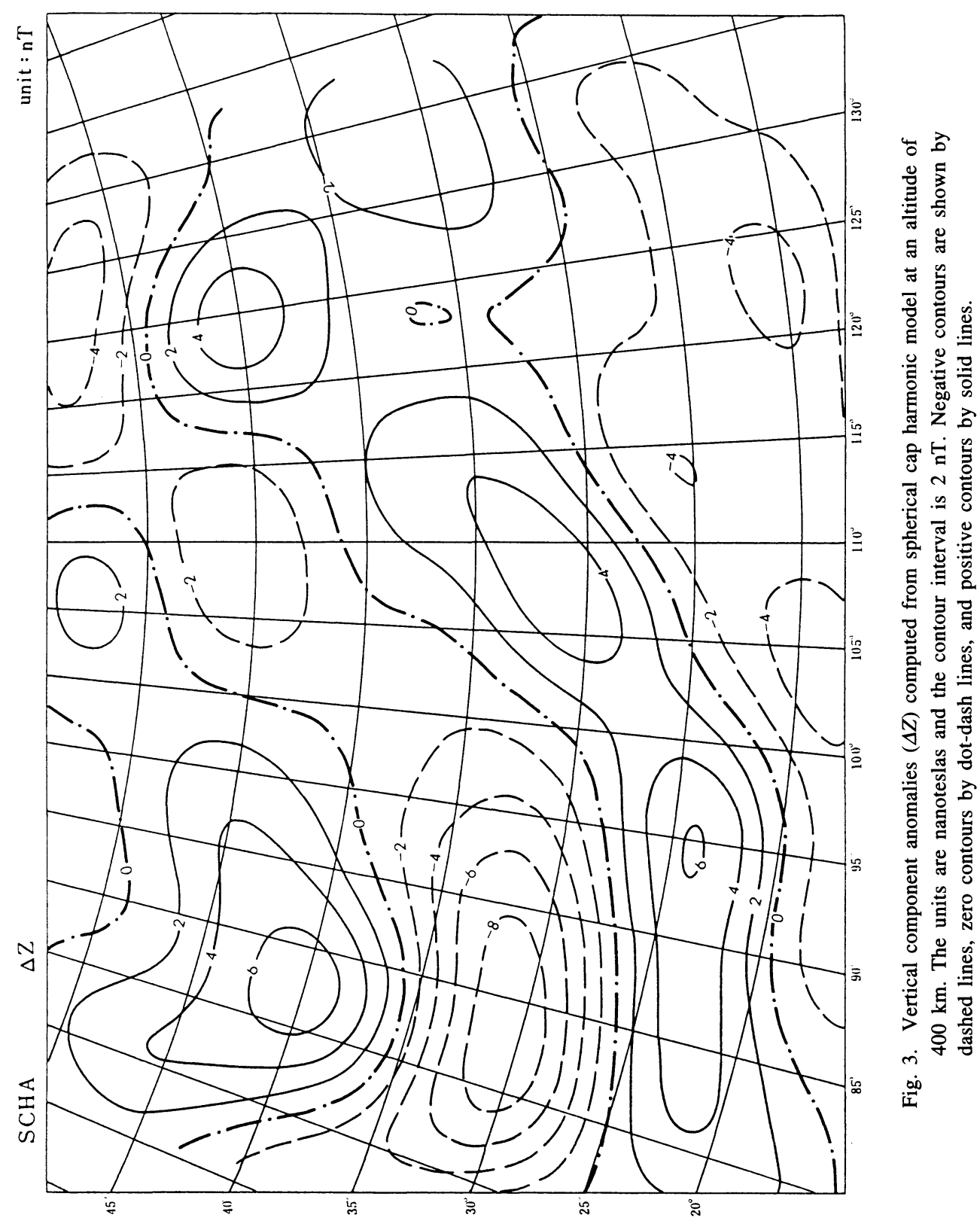




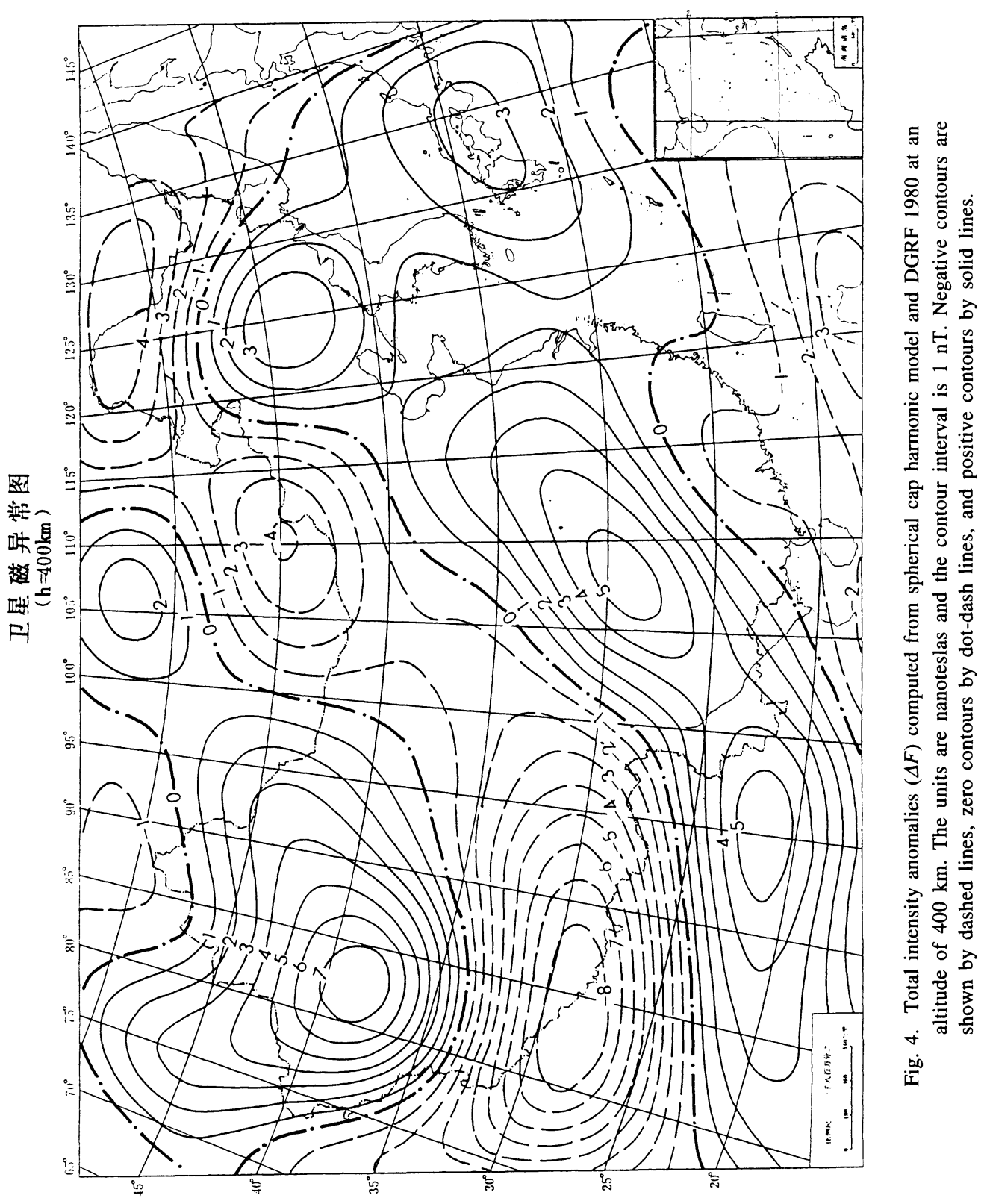


(3) The Heilongjiang-Inner Mongolia-Xizang (Tibet) negative anomaly belt. There are three negative anomaly foci: the first, of intensity $-9 \mathrm{nT}$, in the Himalayan region; the second $(-4 \mathrm{nT})$ on the China-Mongolia border; the third in the Mehe area of Heilongjiang province and $-4 \mathrm{nT}$ in size.

(4) The Baykal-Xinjiang positive anomaly belt, with a $7 \mathrm{nT}$ positive focus in Tarim and one of $2 \mathrm{nT}$ in the region of Lake Baykal.

The satellite magnetic anomalies indicate the lateral variations of crustal magnetisation and show a good correlation with geological and tectonic features. Precambrian blocks such as the Tarim block and the Yangtze block are delineated by positive magnetic anomalies, whilst fold belts, such as the Himalayan and south-east coastal fold belts, and recent uplifts, for example the Tibetan plateau, coincide with negative magnetic anomalies.

\section{Conclusions}

A spherical cap harmonic model of the geomagnetic crustal anomaly field over China and adjacent areas, based on measurements made by the Magsat geomagnetic survey satellite has been described. The model well represents the three-dimensional structure of the anomaly field over this region, certainly for anomalies of medium wavelength (approximately 1000 $\mathrm{km})$.

This research was supported by the National Science Foundation of China (NSFC). It is published with the permission of the Director, British Geological Survey (NERC).

The editor thanks R. A. Langel and M. Yanagisawa for their assistance in evaluating this paper.

\section{REFERENCES}

AN, C. C., S. Z. MA, and D. H. TAN, Spherical cap harmonic analysis of satellite magnetic anomaly in Chinese and adjacent region, Acta Geophys. Sinica, 1992a (in press).

AN, C. C., Y. F. XU, and Y. H. WANG, Magsat vector and scalar magnetic anomaly over Chinese region, Chinese J. Space Sci., 1992b (in press).

Barraclough, D. R., International Geomagnetic Reference Field: the fourth generation, Phys. Earth Planet. Inter., 48, 279-292, 1987.

HaInes, G. V., Spherical cap harmonic analysis, J. Geophys. Res., 90, 2583-2591, 1985.

LANGel, R. A. and R. H. Estes, The near-Earth magnetic field at 1980 determined from Magsat data, $J$. Geophys. Res., 90, 2495-2509, 1985.

Langel, R. A., J. Berbert, T. Jenning, and R. Horner, Magsat data processing: a report for investigators, NASA Tech. Memo., TM82160, 1981.

YIN, S. H., Basic characteristics of the gravity field in China, Seismogeology, 2, 67-75, 1980. 